Provided for non-commercial research and education use. Not for reproduction, distribution or commercial use.

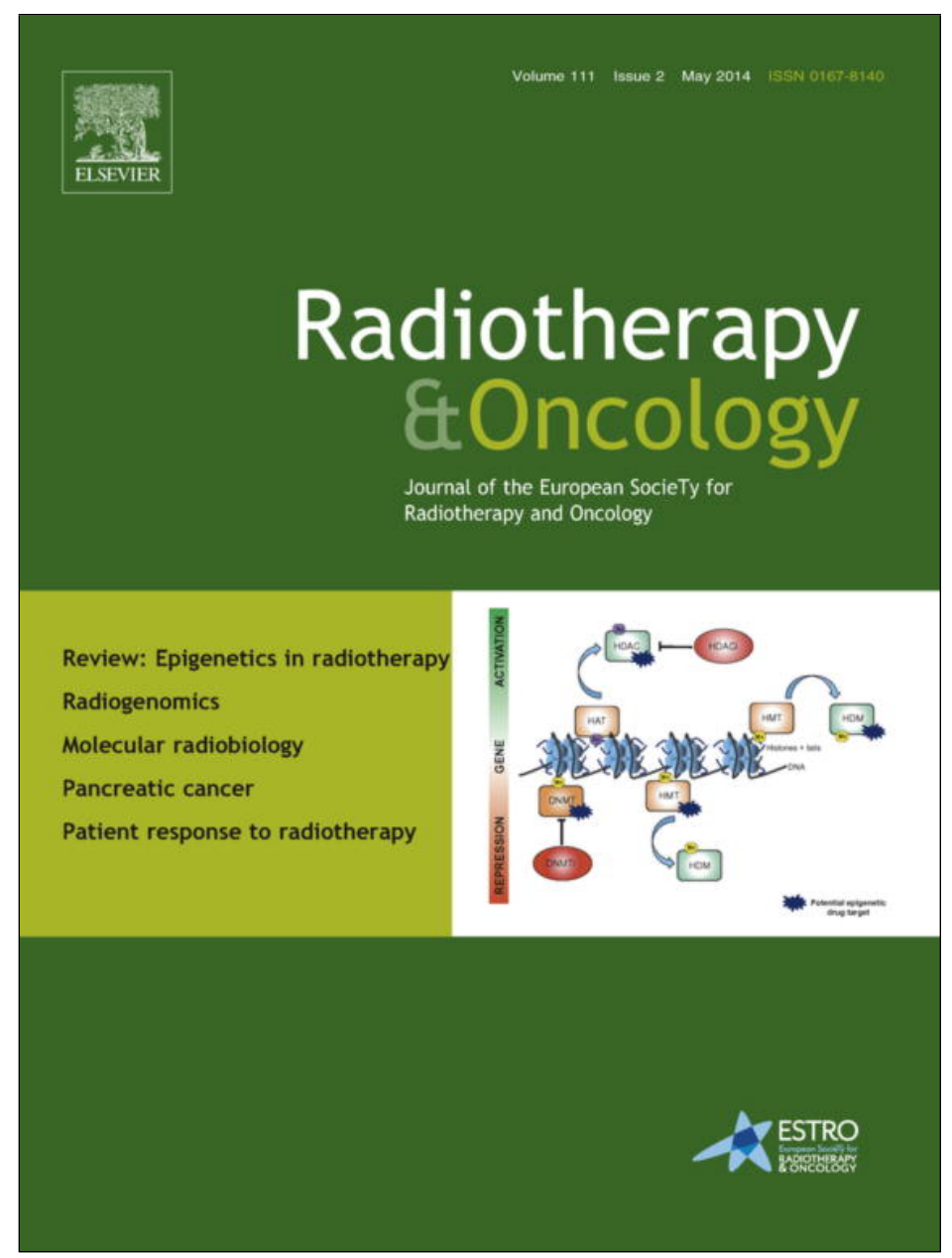

This article appeared in a journal published by Elsevier. The attached copy is furnished to the author for internal non-commercial research and education use, including for instruction at the authors institution and sharing with colleagues.

Other uses, including reproduction and distribution, or selling or licensing copies, or posting to personal, institutional or third party websites are prohibited.

In most cases authors are permitted to post their version of the article (e.g. in Word or Tex form) to their personal website or institutional repository. Authors requiring further information regarding Elsevier's archiving and manuscript policies are encouraged to visit:

http://www.elsevier.com/authorsrights 
Anxiety in breast cancer RT

\title{
Anxiety and its time courses during radiotherapy for non-metastatic breast cancer: A longitudinal study
}

\author{
Florence Lewis $^{\mathrm{a}}$, Isabelle Merckaert ${ }^{\mathrm{a}, \mathrm{b}}$, Aurore Liénard ${ }^{\mathrm{a}, \mathrm{b}}$, Yves Libert ${ }^{\mathrm{a}, \mathrm{b}}$, Anne-Marie Etienne ${ }^{\mathrm{c}}$, \\ Christine Reynaert ${ }^{\mathrm{d}}$, Jean-Louis Slachmuylder ${ }^{\mathrm{e}}$, Pierre Scalliet ${ }^{\mathrm{f}}$, Van Houtte Paul ${ }^{\mathrm{a}, \mathrm{b}}$, Philippe Coucke ${ }^{\mathrm{g}}$, \\ Emile Salamon ${ }^{\mathrm{h}}$, Darius Razavi ${ }^{\mathrm{a}, \mathrm{b}, *}$
}

${ }^{a}$ Université Libre de Bruxelles, Faculté des Sciences Psychologiques et de l'Éducation; ${ }^{\mathrm{b}}$ Institut Jules Bordet, Brussels; ${ }^{\mathrm{C}}$ Université de Liège, Faculté des Sciences Psychologiques et de l'Éducation; ${ }^{\mathrm{d}}$ Université Catholique de Louvain, Faculté de Psychologie et des Sciences de l'Éducation, Louvain-la-Neuve; ${ }^{\mathrm{e}}$ C.P.O. (Centre de Psycho-Oncologie), Brussels, Liège; ${ }^{\mathrm{f}}$ Université Catholique de Louvain, Faculté de Médecine, Brussels; ${ }^{\mathrm{g}}$ Université de Liège, Faculté de Médecine, Liège; ${ }^{\mathrm{h}}$ Clinique Saint-Elisabeth, Namur, Belgium

\section{A R T I C L E I N F O}

\section{Article history:}

Received 14 November 2013

Received in revised form 27 March 2014

Accepted 28 March 2014

Available online 17 April 2014

\section{Keywords:}

Cancer

Anxiety

Radiotherapy

Distress

Screening

\begin{abstract}
A B S T R A C T
Purpose: To our knowledge, no study has specifically assessed the time course of anxiety during radiotherapy (RT). The objective of this study was to assess anxiety time courses in patients with nonmetastatic breast cancer.

Material and methods: This multicenter, descriptive longitudinal study included 213 consecutive patients with breast cancer who completed visual analog scales (VASs) assessing state anxiety before and after the RT simulation and the first and last five RT sessions.

Results: Pre- and post-session anxiety mean levels were highest at the RT simulation (respectively, $2.9 \pm 2.9$ and $1.6 \pm 2.5$ ) and first RT session (respectively, $3.4 \pm 2.9$ and $2.0 \pm 2.4$ ), then declined rapidly. Clinically relevant mean differences ( $\geqslant 1 \mathrm{~cm}$ on the VAS) between pre- and post-simulation/session VAS scores were found only for the RT simulation $(-1.3 \pm 2.7 ; p<0.001)$ and first RT session $(-1.4 \pm 2.4 ; p<0.001)$. Five percent to $16 \%$ of patients presented clinically relevant anxiety (pre- and post-simulation/session VAS scores $\geqslant 4 \mathrm{~cm}$ ) throughout treatment.

Conclusions: To optimize care, RT team members should offer all patients appropriate information about treatment at the simulation, check patients' understanding, and identify patients with clinically relevant anxiety requiring appropriate support throughout RT.
\end{abstract}

(c) 2014 Elsevier Ireland Ltd. All rights reserved. Radiotherapy and Oncology 111 (2014) 276-280
Radiotherapy may generate many fears [1-3] that persist after the start of treatment and despite information transmission [1]. Although some studies have shown that radiotherapy is generally better accepted than what may be expected $[3,4]$ and than other treatments [5], other studies have also shown that it can be associated with emotional distress [6-8] and especially anxiety $[3,5,7,9]$. Ten to twenty percent of patients with cancer experience anxiety before the start of radiotherapy [7] and $20-50 \%$ of patients feel anxious during the first days of treatment; these feelings tend to decrease throughout the course of treatment, although results have varied greatly across studies [7,10,11].

Anxiety related to a given radiotherapy session may be anticipatory or the sign of clinically relevant anxiety [12]. Anticipatory anxiety can be defined as the appearance of anxious symptoms

\footnotetext{
* Corresponding author. Address: Université Libre de Bruxelles, Faculté des Sciences Psychologiques et de l'Éducation, Av. F. Roosevelt, 50 - CP 191, B-1050 Bruxelles, Belgium.

E-mail address: drazavi@ulb.ac.be (D. Razavi).
}

and feelings in the days/hours before a feared event, and their rapid decline after the event. It leads to autonomic arousal and increases vigilance toward the environment [13], and may be experienced by individuals with or without an anxiety disorder [14]. A moderate level of anticipatory anxiety as an emotional signal can lead to defensive and coping reactions, and may enhance adjustment $[5,15,16]$. This form of anxiety, however, can also lead to uncontrolled ruminations and intrusive thoughts [17]. Anticipatory anxiety is thus generally considered to be a normal reaction that may sometimes lead to emotional distress [18] or be a sign of clinically relevant anxiety.

Few studies have focused specifically on the time course of anxiety throughout radiotherapy treatment. One study suggested that anxiety "prior" to radiotherapy is associated with the lack of information about treatment, side effects, and the procedure [2]. Another study suggested that anxiety at the first radiotherapy session is associated with psychological distress and some characteristics of the radiotherapy environment (e.g., waiting room, linear accelerator room), but not with patients' coping strategies, cancer 
prognosis, or experience of other treatments [3]. To our knowledge, no study has specifically assessed the time course of anxiety before and after radiotherapy sessions.

The main objective of this study was to examine anxiety time courses during radiotherapy in patients with non-metastatic breast cancer. Anxiety was measured at the radiotherapy simulation and at each radiotherapy session during the first and last weeks of radiotherapy treatment. Three time courses were considered. The first and second time courses considered anxiety levels just before and after all radiotherapy simulation/sessions, respectively, and the third time course considered the difference in anxiety levels before and after simulation/sessions. Given the "unknown" factor related to the initiation of radiotherapy, we hypothesized that patients would feel high levels of anxiety during the radiotherapy simulation and first week of radiotherapy, and lower levels of anxiety at the start of the last week of radiotherapy. Given the "unknown" factor related to the termination of radiotherapy, we hypothesized that patients' anxiety levels would increase at the last radiotherapy sessions. Given the "unknown" factor related to radiotherapy administration, we hypothesized that the difference in anxiety levels before and after radiotherapy simulation/sessions would be clinically relevant during the first week. As clinically relevant anxiety requires appropriate support, we assessed the prevalence of this condition at different timepoints. We hypothesized that a minority of patients would exhibit clinically relevant anxiety and that this anxiety would remain stable throughout treatment.

\section{Materials and methods}

\section{Subjects and setting}

This study was part of an interuniversity research program conducted in four radiotherapy units in Belgium. The program tested the efficacy of a communication skills training program on radiotherapy team member communication and interdisciplinary work [19]. Radiotherapy team members were invited to participate in the study and asked to provide permission for their patients' inclusion. Local ethics committees approved the study. The results discussed in the present article were based only on patient data. All consecutive patients who fulfilled the inclusion criteria were invited to participate. Women aged $\geqslant 18$ years with surgically treated primary breast cancer without metastasis, who were receiving radiotherapy for the first time, had no cognitive dysfunction, and provided written informed consent were included in the study.

\section{Study design and assessment}

Independent investigators recruited patients for this multicenter descriptive longitudinal study and assisted them in the completion of questionnaires. Patients' anxiety levels were assessed at the radiotherapy simulation and the first and last five radiotherapy sessions, respectively.

\section{Questionnaires}

Sociodemographic data. Patients provided demographic information, including age, marital status, educational level, occupational status, and cultural origin on a questionnaire.

Disease-related characteristics. Physicians provided data about patients' disease and treatment characteristics, including diagnoses (months since diagnosis, disease stage) and received or scheduled treatments (surgery, chemotherapy, radiotherapy, hormonotherapy, biological therapy).

\section{Measurement of anxiety level}

Before and after the radiotherapy simulation, the first five radiotherapy sessions (first week of radiotherapy), and the last five radiotherapy sessions (last week of radiotherapy), patients were asked to report their anxiety levels using a visual analog scale (VAS). The VAS consisted of horizontal $10-\mathrm{cm}$ line, with the extreme left defined as "not at all anxious" and the extreme right defined as "extremely anxious." The VAS was used because such scales have been shown to be appropriate and adequate for the assessment of state anxiety [20-22] and is easy to complete.

The time course of anxiety level before radiotherapy simulation/ sessions was assessed by comparing patients' state anxiety VAS scores reported just before the radiotherapy simulation, first five sessions, and last five sessions of treatment.

The time course of anxiety level after radiotherapy simulation/sessions was assessed by comparing patients' state anxiety VAS scores reported just after the radiotherapy simulation, first five sessions, and last five sessions of treatment.

The time course of the difference in anxiety levels before and after radiotherapy simulation/sessions was assessed by determining the differences in state anxiety VAS scores reported just after and before the radiotherapy simulation, first five sessions, and last five sessions of treatment. As recommended by Mitchell [23,24], we considered patients with both pre- and post-session VAS scores $\geqslant 4 \mathrm{~cm}$ to have clinically relevant anxiety and a difference $\geqslant 1 \mathrm{~cm}$ between pre- and post-session VAS scores to be clinically relevant.

\section{Statistical analyses}

State anxiety VAS scores obtained before and after the radiotherapy simulation and sessions were compared using the Wilcoxon matched-pairs test. One-way multivariate analysis of variance (MANOVA) was used to compare all pre-session and post-session state anxiety VAS scores, respectively. Bonferroni correction was then applied to these multiple comparisons. All tests were two-tailed and the alpha level was set to 0.05 . Analyses were performed with IBM SPSS software (version 19.0; SPSS Inc., Chicago, IL, USA).

\section{Results}

\section{Subjects}

Of 340 consecutive patients approached, 47 (14\%) did not meet inclusion criteria and 47/293 (16\%) eligible patients refused to participate. During the course of the study, 10 (3\%) patients decided to stop the completion of questionnaires. Only patients who completed the state anxiety VAS at the radiotherapy simulation and first and fifth radiotherapy sessions in the first and last weeks of radiotherapy were included. Twenty-three patients were not included in the data analysis. Data from 213 (73\%) patients were analyzed.

\section{Patients' sociodemographic, disease, and treatment characteristics}

All patients included in this study were middle aged (mean, $55 \pm 11$ years; range, $27.9-84.3$ years $) ; 75 \%(n=159)$ were married or cohabiting, $74 \%(n=158)$ had at least high-school education, and $27 \%(n=57)$ worked part or full time. The mean time since diagnosis was 4.4 [standard deviation $(\mathrm{SD})=3.2$ ] months. The majority ( $85 \%, n=181$ ) of patients had stage I or II disease. Most $(78 \%$, $n=167$ ) of them had undergone lumpectomy and about half $(53 \%, n=112)$ had received chemotherapy. The radiotherapy treatment comprised an average of $23(\mathrm{SD}=3.2)$ sessions, and most (71\%, $n=151$ ) patients attended booster sessions. Only $12 \%$ 
$(n=25)$ of patients had received biological therapy (Trastuzumab). Hormonotherapy was scheduled for $70 \%(n=150)$ of patients.

\section{Time courses of anxiety levels}

Table 1 and Fig. 1 display patients' state anxiety VAS scores at the radiotherapy simulation and first and last five radiotherapy sessions.

\section{Anxiety level before radiotherapy simulation/sessions}

Patients' anxiety levels just before sessions were highest at the radiotherapy simulation (mean, $2.9 \pm 2.9$ ) and first radiotherapy session (mean, $3.4 \pm 2.9$ ), then decreased rapidly and dramatically at subsequent sessions in the first week of radiotherapy. These low levels of anxiety persisted during the last week of radiotherapy. MANOVA revealed significant changes $(p<0.001)$ in state anxiety VAS scores before the simulation and first and last five radiotherapy sessions. After Bonferroni correction, only anxiety levels at the simulation and first session differed significantly from those at all other sessions $(p<0.001)$; moreover anxiety levels at the simulation and first session were not significantly different $(p=0.005)$

\section{Anxiety level after radiotherapy simulation/sessions}

Patients' anxiety levels just after sessions were low at the radiotherapy simulation (mean, $1.6 \pm 2.5$ ) and slightly higher at the first session of treatment (mean, $2.0 \pm 2.4$ ), then decreased at subsequent sessions in the first week of treatment and stabilized through the end of treatment. MANOVA revealed significant changes $(p<0.001)$ in state anxiety VAS scores after the simulation and first and last five radiotherapy sessions. After Bonferroni correction, only anxiety level at the first radiotherapy session differed

Table 1

Time course of anxiety during the simulation and the first and last weeks of radiotherapy (RT): comparisons of anxiety levels just before and just after RT sessions and differences in anxiety levels just before and just after RT sessions.

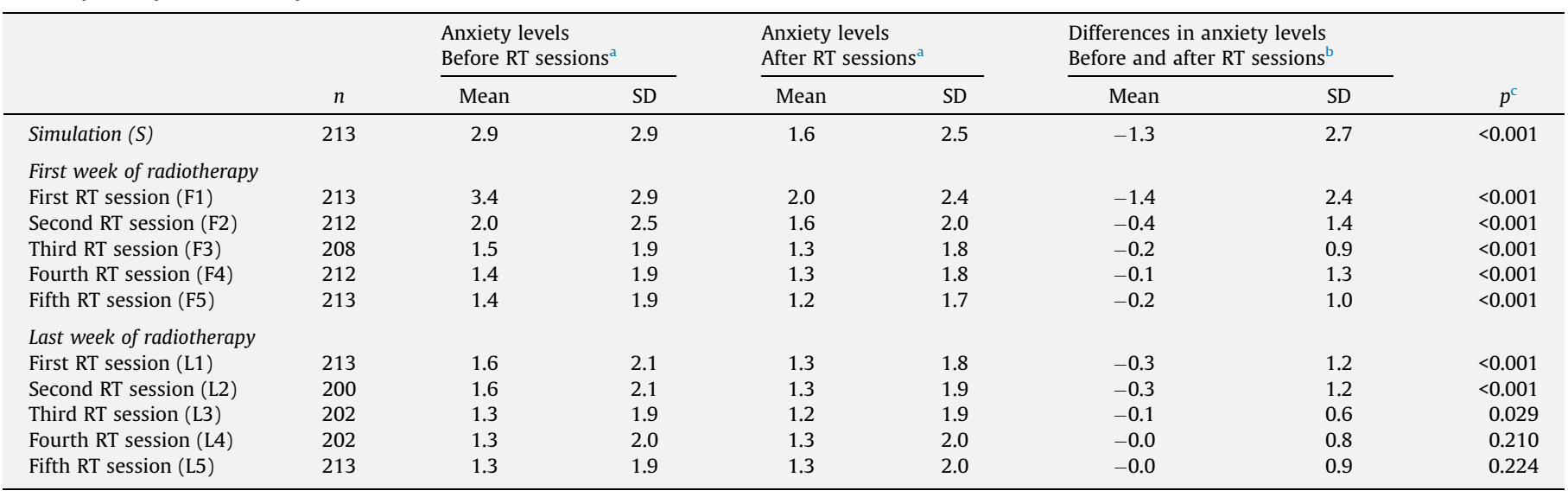

a Measured through state anxiety VAS scores $(\mathrm{cm})$ before and after radiotherapy sessions.

b Measured through the difference between state anxiety VAS scores after and before sessions.

c Wilcoxon matched-pairs test.

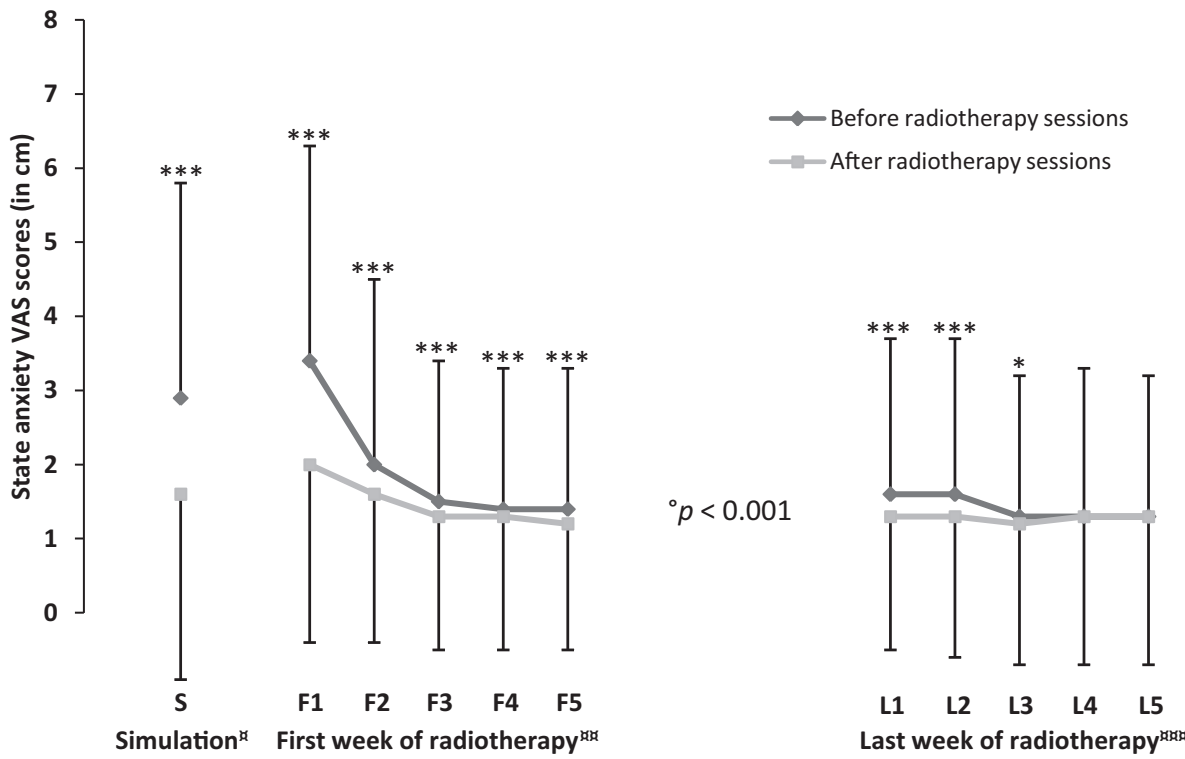

Fig. 1. Time course of anxiety among patients during the simulation and the first and last weeks of radiotherapy (RT). Comparisons of the levels of anxiety before and after each RT session (measured on state anxiety VAS scores) through Wilcoxon matched-pairs tests; ${ }^{* * *} p<0.001,{ }^{*} p<0.05$; ${ }^{\circ}$ MANOVA time changes in state anxiety VAS scores before and after the simulation and sessions; ${ }^{\text {a }}$ S refers to RT simulation; ${ }^{\text {ax }}$ F1 to F5 refer to RT sessions in the first week of RT; ${ }^{\text {and }}$ L1 to L5 refer to RT sessions in the last week of RT. 
significantly from those at all other sessions $(p<0.001)$; moreover anxiety level at the first session was not significantly different from the simulation $(p=0.002)$.

\section{Difference in anxiety before and after simulation/sessions}

Significant differences between pre- and post-session anxiety levels were found at the simulation, all sessions in the first week of radiotherapy, and the first three sessions in the last week of radiotherapy ( $p$ values ranging from $<0.001$ to 0.029 ). Clinically relevant $(\geqslant 1 \mathrm{~cm})$ differences in pre- and post-session VAS scores were found only at the simulation (mean, $-1.3 \pm 2.7$ ) and the first session (mean, $-1.4 \pm 2.4$ ). This difference decreased at the second session (mean, $-0.4 \pm 1.4$ ) and was almost completely absent at the following sessions.

\section{Evolution of clinically relevant anxiety rate over time}

Fig. 2 shows the evolution over time of the rate of patients reporting clinically relevant anxiety (pre- and post-session VAS scores $\geqslant 4 \mathrm{~cm}$ ). At the simulation, $13 \%$ of patients reported clinically relevant anxiety. This rate was highest (16\%) at the first session, then decreased (12\%) at the second session and remained stable (5-10\%) at subsequent sessions in the first and last weeks of radiotherapy. It should be noted that some patients developed clinically relevant anxiety during the course of treatment: 20 (9.4\%) at the first session, 7 (3.3\%) at the second session, $4(1.9 \%)$ at the third session, $1(0.5 \%)$ at the fifth session in the first week of radiotherapy, and $6(2.8 \%)$ at the first session and $1(0.5 \%)$ at the second session in the last week of radiotherapy. No new case of clinically relevant anxiety was observed at the fourth session in the first week of radiotherapy and at the last three sessions in the last week of radiotherapy.

\section{Discussion}

The main objective of this study was to examine anxiety time courses during radiotherapy for breast cancer. The results indicate that anxiety levels decreased rapidly after the radiotherapy simulation and first radiotherapy session in most patients with nonmetastatic breast cancer. A small minority of patients experienced clinically relevant anxiety, and their anxiety levels remained high throughout treatment.
Patients' anxiety levels were highest before and after the simulation and first treatment session. Pre-session anxiety levels were generally higher than post-session levels in the first and last weeks of radiotherapy, but clinically relevant differences (difference $\geqslant 1 \mathrm{~cm}$ between pre- and post-session VAS scores) were found only for the simulation and first session. Contrary to our hypotheses and previous findings [1], we observed a rapid decline in anxiety levels after the first radiotherapy session. This may be explained by the alleviation of many patients' fears of the unknown and of potential side effects (e.g., damage to internal organs, burns, pain, fatigue) before starting radiotherapy and in the first days of treatment due to the absence of perceived side effects during the first session. The rapid decrease in anxiety levels thus reflects patients' habituation to treatment, which may have been mediated by support provided by the radiotherapy team.

Contrary to our hypotheses, patients' anxiety levels did not increase during the last days of radiotherapy, which may be explained by patients' relief at the end of lengthy radiotherapy treatment. Anxiety levels might increase later, when patients face concerns about their futures, such as those regarding the persistence of physical symptoms, fear of recurrence, and return to work. Anxiety levels measured during radiotherapy are lower than those measured during other treatments, such as chemotherapy [5]. Thus, the results of the present study reflect a normal reaction which seems to be mainly anticipatory as it decreases.

Although most patients' anxiety levels were low and decreased rapidly after the first session, $5-16 \%$ of patients presented clinically relevant anxiety (pre- and post-simulation/session VAS scores $\geqslant$ $4 \mathrm{~cm}$ ) during treatment. This proportion corresponds to the prevalence of anxiety disorders in the general population (10-17\%) $[25,26]$. For these patients, anxiety was not only a normal anticipatory reaction, but the sign of a clinically relevant anxiety. These patients maintained disturbing levels of anxiety throughout radiotherapy. In other words, these patients have a clinically relevant anxiety separate from what is experienced during treatment. Their levels of anxiety are thus not affected by habituation to treatment.

This study has several strengths. To our knowledge, it is the first investigation of the time courses of anxiety before and after simulation/treatment sessions and throughout treatment. Second, patients' anxiety levels were measured repeatedly using the VAS, a valid measure that is sensitive to change. Some limitations of the study should also be mentioned. First, patients were assessed

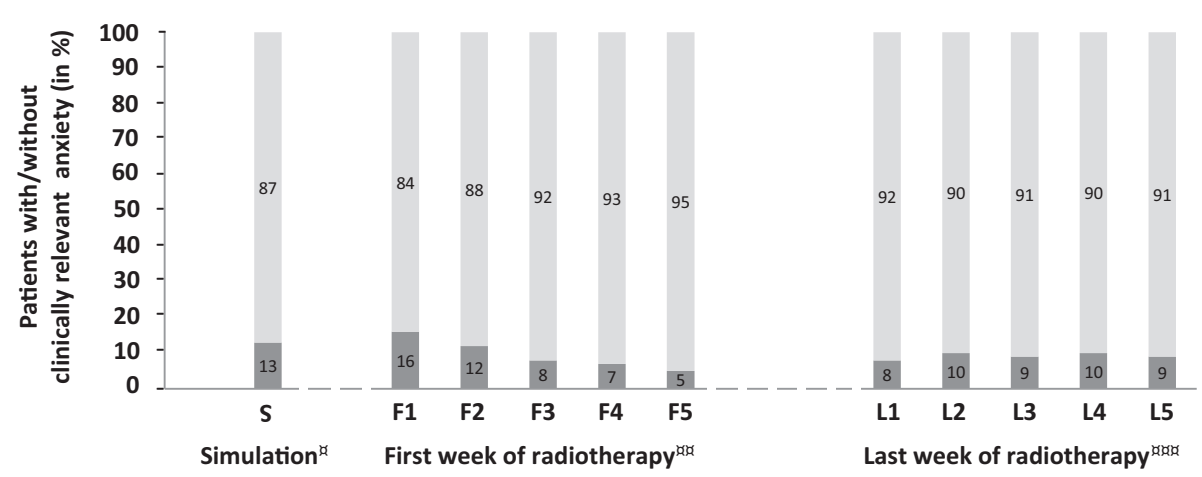

Fig. 2. Evolution over time of the rate of breast cancer patients with clinically relevant anxiety (pre- and post-simulation/session VAS scores $\geqslant 4 \mathrm{~cm}$ ) and without clinical anxiety (pre- and/or post-simulation/session $<4 \mathrm{~cm}$ ). ${ }^{\text {a }} \mathrm{S}$ refers to radiotherapy (RT) simulation; ${ }^{\text {ax }} \mathrm{F} 1$ to F5 refer to RT sessions in the first week of RT; ${ }^{\text {and }}$ L1 to L5 refer to RT sessions in the last week of RT. 
only during the first and last weeks of radiotherapy treatment. Thus, our results do not reflect their psychological functioning during the entire course of treatment or after its conclusion. Second, our sample included only patients with non-metastatic breast cancer and the results may not be generalizable to other populations of patients with cancer.

The results of this study allow us to make two recommendations to radiotherapy team members regarding the care of patients with non-metastatic breast cancer. First, team members should be aware that patients' anxiety levels peak at the simulation and first treatment session due to fear of the unknown. Team members should thus provide all patients with appropriate oral and written (e.g., pamphlets, digital resources) information about radiotherapy (e.g., procedures, side effects) at the simulation, and systemically check patients' comprehension and correct misunderstandings when necessary. Second, team members should be aware that patients may present clinically relevant anxiety requiring appropriate support throughout radiotherapy. Team members should thus evaluate patients' anxiety as early as possible using brief, effective screening tools (e.g., VAS) that assess emotional states in general and anxiety levels in particular. Such assessment is not time consuming and can readily identify patients who may require appropriate support and referral to mental health professionals for personalized treatment. Ideally, team members should investigate patients' anxiety no later than the radiotherapy simulation, so that they can implement support during radiotherapy as appropriate. Team members should repeat this assessment of patients' anxiety each week of treatment to detect new cases of patients presenting clinically relevant anxiety. Brief training of team members may facilitate the implementation of these recommendations.

Although these results improve the understanding of the time course of patients' anxiety during radiotherapy and may be useful for the organization of clinical care, further investigation of the predictors of anxiety (sociodemographic, medical, psychological, and social variables) is needed. Importantly, further research is also needed to evaluate the efficacy of support provided by team members to patients throughout treatment, including the ways in which they inform patients and early referrals to mental health professionals for appropriate support.

\section{Role of the funding source}

The study sponsors had no role in the study design or data collection, analysis, or interpretation; or in the preparation, review, or approval of the manuscript.

\section{Conflict of interest}

The authors have no financial or personal relationships with individuals or organizations that could inappropriately influence their work. The principal investigator had full access to all study data and takes responsibility for the integrity of the data and accuracy of the analysis.

\section{Acknowledgements}

This study was supported by the "Fonds National de la Recherche Scientifique - Télévie" of Belgium (credit number: FC 51034/7.4.538.07 F) and the Centre de Psycho-oncologie of Brussels. The authors would like to thank the participating hospitals and radiotherapy teams: the "Institut Jules Bordet ULB" in Brussels (Professor Paul Van Houtte); the "Cliniques universitaires Saint-Luc" in Brussels (Professor Pierre Scalliet); the
"Clinique Saint-Elisabeth" in Namur (Professor Emile Salamon) and the "Centre Hospitalier Universitaire" of Liège (Professor Philippe Coucke). The authors also thank the participating patients.

\section{References}

[1] Halkett GK, Kristjanson LJ, Lobb EA. 'If we get too close to your bones they'll go brittle': women's initial fears about radiotherapy for early breast cancer. Psychooncology 2008;17:877-84.

[2] Halkett GK, Kristjanson LJ, Lobb E, et al. Information needs and preferences of women as they proceed through radiotherapy for breast cancer. Patient Educ Couns 2012:86:396-404.

[3] Mose S, Budischewski KM, Rahn AN, Zander-Heinz AC, Bormeth S, Bottcher HD. Influence of irradiation on therapy-associated psychological distress in breast carcinoma patients. Int J Radiat Oncol Biol Phys 2001;51:1328-35.

[4] Wallace LM, Priestman SG, Dunn JA, Priestman TJ. The quality of life of early breast cancer patients treated by two different radiotherapy regimens. Clin Oncol (R Coll Radiol) 1993;5:228-33.

[5] Lim CC, Devi MK, Ang E. Anxiety in women with breast cancer undergoing treatment: a systematic review. Int J Evid Based Healthc 2011;9:215-35.

[6] Fritzsche K, Liptai C, Henke M. Psychosocial distress and need for psychotherapeutic treatment in cancer patients undergoing radiotherapy. Radiother Oncol 2004;72:183-9.

[7] Stiegelis HE, Ranchor AV, Sanderman R. Psychological functioning in cancer patients treated with radiotherapy. Patient Educ Couns 2004;52:131-41.

[8] Schnur JB, David D, Kangas M, Green S, Bovbjerg DH, Montgomery GH. A randomized trial of a cognitive-behavioral therapy and hypnosis intervention on positive and negative affect during breast cancer radiotherapy. J Clin Psychol 2009;65:443-55.

[9] Hopwood P, Haviland J, Mills J, Sumo G. J MB. The impact of age and clinical factors on quality of life in early breast cancer: an analysis of 2208 women recruited to the UK START Trial (Standardisation of Breast Radiotherapy Trial). Breast 2007; 16:241-51

[10] Leopold KA, Ahles TA, Walch S, et al. Prevalence of mood disorders and utility of the PRIME-MD in patients undergoing radiation therapy. Int J Radiat Oncol Biol Phys 1998;42:1105-12.

[11] Rahn AN, Mose S, Zander-Heinz A, et al. Influence of radiotherapy on psychological health in breast cancer patients after breast conserving surgery. Anticancer Res 1998;18:2271-3.

[12] Hopwood P, Sumo G, Mills J, Haviland J, Bliss JM. The course of anxiety and depression over 5 years of follow-up and risk factors in women with early breast cancer: results from the UK Standardisation of Radiotherapy Trials (START). Breast 2010;19:84-91.

[13] Gray J. The neuropsychology of anxiety: an enquiry into the functions of the septo-hippocampal system. New York: Oxford University Press; 2000. 440.

[14] Barlow DH, Chorpita BF, Turovsky J. Fear, panic, anxiety, and disorders of emotion. Nebr Symp Motiv 1996;43:251-328.

15] Janis JL, editor. Psychological stress: Psychoanalytic and behavioral studies of surgical patients. New York: Wiley; 1958.

[16] Andersen BL, Tewfik HH. Psychological reactions to radiation therapy: reconsideration of the adaptive aspects of anxiety. J Pers Soc Psycho 1985;48:1024-32.

[17] Vassilopoulos SP. Anticipatory processing in social anxiety. Behav Cogn Psychother 2004;32:303-11.

[18] Vassilopoulos SP. Coping strategies and anticipatory processing in high and low socially anxious individuals. J Anxiety Disord 2008;22:98-107.

[19] Razavi D, Coucke P, Scalliet P, et al. L'optimalisation de la réponse aux besoins psychologiques des patientes traitées pour une affection cancéreuse du sein par un programme de formation à la communication destinée aux équipes de radiothérapie: une étude randomisée. Bruxelles: Projet interuniversitaire; 2005.

[20] Wewers ME, Lowe NK. A critical review of visual analogue scales in the measurement of clinical phenomena. Res Nurs Health 1990;13:227-36.

[21] Miller MD, Ferris DG. Measurement of subjective phenomena in primary care research: the Visual Analogue Scale. Fam Pract Res J 1993;13:15-24.

[22] Davey HM, Barratt AL, Butow PN, Deeks JJ. A one-item question with a Likert or Visual Analog Scale adequately measured current anxiety. J Clin Epidemiol 2007:60:356-60.

[23] Mitchell AJ, Baker-Glenn EA, Granger L, Symonds P. Can the Distress Thermometer be improved by additional mood domains? Part I. Initial validation of the Emotion Thermometers tool. Psychooncology 2010;19:125-33.

[24] Mitchell AJ, Baker-Glenn EA, Park B, Granger L, Symonds P. Can the Distress Thermometer be improved by additional mood domains? Part II. What is the optimal combination of Emotion Thermometers? Psychooncology 2010;19:134-40.

[25] Somers JM, Goldner EM, Waraich P. Hsu L. Prevalence and incidence studies of anxiety disorders: a systematic review of the literature. Can J Psychiatry 2006;51:100-13.

[26] Baxter AJ, Scott KM, Vos T, Whiteford HA. Global prevalence of anxiety disorders: a systematic review and meta-regression. Psychol Med 2013;43:897-910. 